\title{
Agôn
}

Revue des arts de la scène

$5 \mid 2012$

L'entrée en scène

\section{Hanté en scène : avant le geste et avant le son. L'entrée en scène du musicien}

Entretien réalisé par Jean-François Trubert

Philippe Serra et Jean-François Trubert

\section{(2) OpenEdition}

\section{Journals}

Édition électronique

URL : http://journals.openedition.org/agon/2430

DOI : 10.4000 /agon.2430

ISSN : 1961-8581

Éditeur

Association Agôn

Référence électronique

Philippe Serra et Jean-François Trubert, « Hanté en scène : avant le geste et avant le son. L'entrée en scène du musicien », Agôn [En ligne], 5 | 2012, mis en ligne le 21 janvier 2013, consulté le 15 septembre 2020. URL : http://journals.openedition.org/agon/2430

Ce document a été généré automatiquement le 15 septembre 2020.

Association Agôn et les auteurs des articles 


\section{Hanté en scène : avant le geste et avant le son. L'entrée en scène du musicien}

Entretien réalisé par Jean-François Trubert

Philippe Serra et Jean-François Trubert

\section{NOTE DE L'ÉDITEUR}

Entretien réalisé le 13 septembre 2012, annexe de l'article « Hanté en scène : avant le geste et avant le son dans l'interprétation musicale et le théâtre musical et instrumental ».

Jean-François trubert : Pour commencer je voudrais que vous nous rappeliez quelques détails biographiques et votre position actuelle, votre profession et les étapes marquantes de votre carrière d'interprète.

Philippe SERRA : Je m'appelle Philippe Serra, j'ai 50 ans et je suis né à Marseille. Je suis artiste-musicien, timbalier-solo de l'orchestre philharmonique de Nice depuis 1989 . J'ai étudié la musique au conservatoire d'Avignon, puis je suis rentré au Conservatoire National Supérieur de Musique de Paris. J'ai obtenu un premier prix de percussion et un premier prix de musique de chambre, et ensuite j'ai intégré l'orchestre de Nice sur concours. J'ai eu la chance de jouer avec des chefs tels que Pierre Boulez, Léonard Bernstein sur Paris, et d'être invité au sein des deux orchestres de Radio France sous la direction de Myung-Whun Chung et de partir en tournée avec le Philarmonique de Radio France. J'ai pu également effectuer des tournées avec l'orchestre de Nice au Japon et en Europe, et j'ai interprété des pièces en solo pour des festivals comme le festival MANCA de Nice.

JFT : Quelles pièces solos?

PS : Psappha de Xenakis, et on m'a invité pour jouer les Pièces pour timbales de Elliott Carter. Ma spécialité, ce sont les timbales. 
JFT: Comment définiriez-vous les différentes étapes qui caractérisent l'interprétation musicale avant le concert?

PS : Il y a plusieurs étapes. La première consiste en la préparation de l'œuvre, la répétition avec l'orchestre et le travail technique personnel : le choix des baguettes et la documentation éventuelle. Il y a une deuxième étape quelques jours avant le concert ou le jour même, où on a besoin d'une relecture de la partition, d'une concentration particulière, d'une écoute au dernier moment, et puis vient le jour du concert, avec tout le rituel de l'habillage, du costume, et ensuite une dernière étape qui est celle du contact avec l'instrument: une première phase pour s'accorder et pour définir les derniers réglages, et ensuite, l'entrée en scène avec l'orchestre.

JFT : Et pour vous, cette entrée en scène peut-elle être décomposée ? Y aurait-il un moment un peu plus particulier dans cet intervalle, lorsque l'on monte sur le plateau par exemple, ou bien lorsque l'on est en attente juste avant le jeu?

PS : Ce qui est important c'est surtout la prise de contact avec l'instrument. La prise de conscience de l'environnement également, surtout lorsque l'on se trouve dans une nouvelle salle. Tout ceci se fait au moment où l'on s'assoit et où l'on s'accorde avec l'orchestre. Il y a toute une ambiance que l'on essaye de capter au maximum, tout en étant concentré et en essayant de positiver le moment où l'on va jouer.

JFT: Est-ce qu'à votre avis l'entrée en scène du musicien est différente de celle qui caractérise celle du comédien, et pourquoi le serait-elle selon vous?

PS : Pour moi, c'est à peu près pareil, surtout lorsque l'on joue pour la première fois une œuvre, qui est un peu comme une prise de rôle. On est dans un état de concentration extrême, et la tension est plus intense avant d'entrer en scène. On a plus de charge émotionnelle, au même titre qu'un comédien. Par contre, lorsque l'on joue une pièce familière, l'entrée en scène se fait beaucoup plus facilement, de façon plus décontractée.

JFT : Parce qu'on la connaît mieux?

PS : Oui, parce qu'on l'a répétée plusieurs fois. L'entrée en scène est à chaque fois différente. Elle est plus souple, plus fluide. Alors que si c'est la première fois, on a forcément une charge émotionnelle plus importante, une concentration plus intense, une tension.

JFT : À votre avis, quel serait le moment le plus important de l'entrée en scène : le moment où on apparaît sur le plateau, ou le moment qui serait juste avant la première frappe (si on est d'accord pour considérer l'entrée en scène comme l'intervalle constitué par le moment où l'on apparaît à la vue du spectateur et celui on l'on va se mettre à jouer)?

PS : En principe tout est important. Quand on doit exécuter une œuvre, on a réglé tout le rituel, on s'est préparé. Le moment le plus important, c'est celui où l'on va jouer. Mais c'est vrai que l'apparition sur le plateau est un moment où l'on est chargé d'ondes positives, où on s'est conditionné de façon optimale. Mais celui qui précède le jeu reste le plus important.

JFT : Est-ce que vous pensez que ce moment de l'entrée en scène possède plusieurs espaces, en tout cas des espaces émotionnellement différenciés, en partant des loges, coulisses, plateau, surface près de l'instrument?

PS : Je dirais que dès l'arrivée au théâtre, il y a déjà un rituel, chaque musicien en a un : que ce soit dans les loges, ou au moment où il s'échauffe: celui qui répète sa gamme, celui qui pose des sons. Personnellement, je m'habille et ensuite je vais m'accorder le plus vite possible pour être sûr que les timbales sont bien réglées, que 
les baguettes sont bien posées. Ensuite on ressort et on attend l'entrée en scène dans les coulisses, on s'échauffe les poignets, on discute un petit peu, pour se décontracter, cela se déroule dans plusieurs lieux.

JFT : Comment décririez-vous ce moment de la prise de contact avec l'instrument, ce moment où l'on arrive autour de la surface de l'instrument qui est déjà là, qui est fixe ? Lors de ce passage dans cet espace particulier, y a-t-il un changement qui s'opère, quelque chose qui se produit à ce moment-là?

PS : Oui, surtout pour moi qui suis timbalier et qui n'ai pas l'occasion d'avoir les instruments près de moi en permanence, en coulisse ou en loge. C'est un moment important, on a hâte d'arriver sur l'instrument, de le toucher, de l'accorder. C'est un moment de grande sérénité en fait, où on est très calme, on a besoin d'être proche de l'instrument, où on va optimiser vraiment les derniers réglages de son, l'accord, le positionnement sur scène du tabouret, et psychologiquement où on va positiver, regarder les différents traits de la partition brièvement, très calmement.

JFT: J'ai l'impression que les attitudes que vous décrivez pourraient être aisément rapprochées de celles qui caractérisent un sportif avant une compétition : vous donnez à percevoir un événement très technique, qui ressemble à l'idée que l'on se fait d'un athlète faisant le vide dans sa tête avant de courir un sprint.

PS : Oui, c'est un peu ça. C'est un moment où on est en relation avec soi-même et son instrument. Donc il faut savoir exactement ce que l'instrument peut donner. À titre de comparaison, je préfère penser aux bassonistes qui sont toujours en train de tailler des anches, ce sera le moment où ils choisissent leurs dernières anches. Nous c'est le moment où on fait nos derniers réglages, l'accord doit être absolument impeccable. Et puis on a besoin de ce contact avec l'instrument parce qu'on a été un petit peu sevré, c'est un moment où on est seul avec soi-même et avec lui, où on sait qu'on va passer une heure, une heure et demie avec, c'est un moment important.

JFT : Après ce moment de réglage, il y a un petit temps de silence qui va précéder le début du morceau proprement dit.

PS : On ressort après. Puis tout l'orchestre rentre en scène, je suis de nouveau en contact avec l'instrument, et à ce moment-là vient ce silence après l'accord de l'orchestre, où tout l'orchestre se concentre, à l'unisson si je puis dire.

JFT : Et là qu'est-ce qui se passe émotionnellement, on essaye de se concentrer, de rentrer déjà dans le morceau, est-ce qu'on essaye de faire le contact avec l'œuvre qui va se dérouler?

PS : Il y a une espèce de concentration qui fait que l'on doit aborder le concert pendant ce silence, et que l'on doit laisser entrer l'œuvre comme si on ne l'avait jamais entendue, en fait comme si on ne l'avait jamais jouée. Il y a une espèce de relaxation, de sérénité, pour découvrir une œuvre ou pour la faire découvrir, pour y entrer comme si c'était la première fois qu'on l'interprétait. Dans tous les cas on pense au public qui va entendre cette interprétation pour la première fois, donc on capte un peu de son silence, et puis le nôtre, notre propre concentration dans l'orchestre.

JFT : Avant de jouer une pièce, doit-on forcément imaginer toutes ses étapes structurelles ? Va-t-on la jouer avec une certaine dynamique, avec un certain état intérieur? Peut-on se mettre en colère par exemple pour jouer une pièce ? Une fois qu'on joue peut-on passer par d'autres étapes?

PS : C'est la pièce qui va diriger cela. La relaxation c'est primordial, c'est quelque chose qu'il faut absolument avoir pour sortir n'importe quel son, respirer 
évidemment pour ne pas être crispé, pour avoir une jolie attaque même si c'est une attaque forte, justement pour qu'elle ne soit pas agressive. Dès la première attaque il faut déjà être décontracté, tout en étant imprégné de ce que l'on va jouer. Que ce soit dès le début très fort ou dès le début très doux, il faut de toute façon être détendu. Je pense que tout l'orchestre respire. Alors après - si ce sont les violons qui démarrent on écoute ce qui est exposé, et on arrive nous-mêmes à rentrer, lorsque c'est le moment où l'on joue, dans le caractère de l'œuvre.

JFT : Diriez-vous que lorsqu'un interprète joue il est quelqu'un d'autre ? Qu'il est ailleurs, qu'il est un autre ou absorbé? Quelle est pour vous la meilleure expression pour qualifier cet état de l'interprétation musicale?

PS : L'interprète est un professionnel, donc il est habitué à interpréter tous les styles de musique, il a été formé pour cela, quelque soit le style de l'œuvre, l'époque. C'est vrai qu'il est absorbé, moi je dirais qu'il est habité, l'interprétation doit toujours être vivante. Il faut qu'il soit juste, c'est-à-dire qu'il ne faut pas qu'il sur-joue ou qu'il ne joue pas. Il est quelqu'un d'autre, mais sans vouloir être quelqu'un d'autre : il faut qu'il soit vraiment habité par la musique. C'est l'équilibre le plus dur à trouver : être bien dans la musique, ne pas sur-jouer, et ne pas se cantonner dans le savoir-faire et dans le savoir-être au moment où l'on joue. Pour nous les timbaliers c'est très facile de tomber dans le mimétisme, dans des grands gestes qui ne correspondent pas à la sonorité. Je cherche toujours à être dans la musique, c'est elle qui doit me guider. Quelques mesures avant mon jeu, je m'en imprègne pleinement pour m'y sentir bien.

JFT: Quand vous dites que vous êtes habité, est-ce au moment où vous jouez, ou bien juste un peu avant ce moment?

PS : Je pense que c'est juste un peu avant. Au moment où on joue, on est forcément concerné, mais ce qui est important c'est ce qui nous amène au jeu. Dans une pièce symphonique, la partie de timbale n'est pas indépendante de ce qui se passe avant, de ce qui se passe après, donc il faut se concentrer quand même juste avant de jouer. Maintenant pour une œuvre soliste, il y a toute la préparation de l'œuvre en scène, et une fois qu'on est sur scène c'est évidemment au moment où l'on joue que l'on doit être habité, mais bon, c'est peut-être plus facile parce que c'est une œuvre complètement soliste. Mais dans une œuvre orchestrale, bien souvent, on attend quelques mesures et c'est juste avant de jouer qu'il faut vraiment être dedans.

JFT : Pour une œuvre soliste, c'est plus facile parce que vous êtes seul ?

PS : Tout dépend de nous. Dans l'œuvre soliste, comme dans un monologue, tout dépend de nous. Il y a l'entrée en scène, le travail en amont, mais après je n'ai pas de responsabilités vis-à-vis du chef, vis-à-vis des violons, vis-à-vis des trompettes qui joueraient les mêmes rythmes que moi, je joue ma pièce comme je l'entends, j'ai une plus grande liberté. À l'orchestre, on a quand même moins de liberté.

JFT: Dans ces œuvres solo, au moment où vous commencez la pièce, juste avant le premier coup de timbale, est-ce que suivant la pièce, cela nécessite un état émotionnel particulier, différent? J'imagine qu'entre Xenakis et Carter, cela ne demande pas forcément la même disponibilité du corps, et est-ce que cet enjeu se détermine juste avant le premier coup?

PS : Quand on joue Xenakis ${ }^{1}$, il faut quand même avoir une charge émotionnelle assez importante juste avant le premier coup. Là, on est un autre avant de jouer la première note. Il faut plus de spontanéité dans le jeu quand on joue une pièce soliste comme cela. 
JFT: Pourquoi particulièrement la musique de Xenakis entraînerait quelque chose de particulier? Est-ce qu'il y a une écriture particulière dans cette pièce qui peut entraîner chez l'interprète une certaine mise en disponibilité ?

PS : Oui, parce que ce sont des pièces très dynamiques, on retrouve cela dans le Sacre du printemps, dans la danse sacrale. Ce sont des pièces très puissantes donc on est émotionnellement très impliqué pour faire sonner toutes ces pièces. Ce sont des pièces très fortes qui demandent aussi une endurance. Mais en même temps il faut beaucoup de spontanéité pour les jouer.

JFT : Quand vous les jouez, avez-vous toujours conscience du temps qui passe ? Ou vous arrive-t-il parfois d'exécuter une pièce dans sa longueur et puis finalement d'en arriver au bout sans avoir perçu l'écoulement du temps ?

PS : Oui. Il y a une notion du temps qui est complètement différente quand on est très concentré sur une pièce. On est vraiment dans ce que l'on fait, ou comme je le disais, habité par la musique qui est en train de naître de la partition. Le facteur temps n'est plus le même. On a vraiment l'impression parfois que cela a duré 15 secondes.

JFT : Est-ce qu'il y a une expérience particulière qui vous semblerait pertinente de rajouter dans le cadre de cette réflexion, ce qui vous semble vraiment important dans cette idée de l'entrée en scène de l'interprète?

PS : Je pensais à différentes pièces avant l'entrée en scène. Par exemple la première de Brahms : c'est une entrée en scène difficile, même si on l'a jouée plusieurs fois ${ }^{2}$, parce que dès les premières mesures elle développe et elle dégage une puissance dramatique assez incroyable, tellement forte que - même si on connaît cette pièce on n'est jamais sûr de ce que l'on va déployer pour notre entrée en scène. En effet, d'emblée les timbales donnent le ton d'un élan très dramatique et lyrique, et il faut veiller à sa sonorité sans être crispé tout en déployant une force énorme. Dès les premiers instants, pour la sonorité, le caractère, la densité, il ne faut pas être agressif et il faut être très concentré. Voilà une entrée en scène très particulière. J'ai aussi à l'esprit la neuvième de Beethoven, pour l'importance de la partie de timbale, qui nécessite une endurance sur toute l'œuvre, si bien qu'à la fin de cette pièce, on me dit parfois que c'est un concerto pour timbales! On peut encore citer la Deuxième symphonie de Mahler, qui a une charge émotionnelle constante, qui trouve son apogée dans le chœur final, et puis le Sacre du printemps de Stravinsky, pour son énergie, car dans le jeu du timbalier, il y a une précision rythmique sur la fin mais aussi sur toute l'œuvre qui est presque diabolique. Ce sont des entrées en scène qui sont bien marquantes.

JPT : Parce qu'elles nécessitent ce que vous avez décrits précédemment : relaxation mais concentration en même temps, charge émotionnelle importante, en fait une projection dans l'œuvre déjà ?

PS : Oui c'est cela, pour différentes raisons. Soit parce qu'il faut de suite être dedans, soit parce que c'est toute la pièce qui va être très dense, soit parce qu'il va falloir dégager un caractère particulier. Il peut arriver de devoir aller chercher une force en soi pendant toute la soirée : c'est le cas par exemple pour le Sacre du printemps dont j'ai parlé mais aussi pour l'opéra Elektra de Richard Strauss : la partie de timbale doit être à la hauteur du drame et de la complexité instrumentale. 
JPT : En tant que timbalier d'orchestre même en pièce solo, vous avez déjà exécuté des pièces que l'on rattache au théâtre musical contemporain?

PS : J'ai joué une pièce humoristique qui est un clin d'œil au théâtre musical. C'est une pièce pour quatre percussionnistes sur un seul marimba, Martian Tribe (1995) de Emmanuel Séjourné, où il y a une mise en scène décrite, mais l'entrée des interprètes y est complètement libre et spontanée ${ }^{3}$. Cette impression de liberté en entrant sur scène c'était assez exaltant et assez nouveau pour moi. J'ai souvent entendu JeanPierre Drouet, ou d'autres percussionnistes dans des pièces de Kagel ou d'Aperghis et c'est vraiment un jeu qui m'impressionne.

JPT : Est-ce qu'à I'heure actuelle vous pensez que l'on peut faire une différence entre exécution et interprétation?

PS : Pour moi les deux sont liées, sinon il n'y a pas de musique. Je ne vois pas de différence.

JFT : J'ai entendu que vous faisiez beaucoup référence à la préparation d'une œuvre. Audelà de l'aspect technique, il y a un aspect préparatoire c'est-à-dire une compréhension du texte musical?

PS: Dans la préparation, j'inclus la préparation physique pour être en pleine possession de ses moyens techniques, la connaissance de la partition, le travail de l'interprétation selon le genre, les époques, le choix des baguettes. Mais il y a aussi, « intellectuellement », une recherche de documentation sur l'auteur et sur l'œuvre en elle-même. On ne fait pas cela pour toutes les œuvres, mais pour certaines, quand on aborde Wagner par exemple, il est intéressant de connaître les Leitmotivs ${ }^{4}$ surtout quand on va passer six heures dans la fosse ${ }^{5}$. Dans les symphonies de Gustav Mahler également, pour l'interprétation, je trouve que c'est bien de savoir si ce sont des fortissimos d'exaltation ou si ce sont des fortissimos dramatiques. C'est important de savoir ce qu'il y a derrière une œuvre, quand on aborde l'écriture de la pièce dans son ensemble. Je regarde souvent la partie de timbale à travers la partie d'orchestre pour bien comprendre l'architecture de la pièce et en faire son analyse.

\section{NOTES}

1. Ici, Philippe Serra fait référence à la pièce solo Psappha (1975) de Iannis Xenakis, solo de percussion pour cinq groupes de percussions non déterminées.

2. Johannes Brahms, 1ère symphonie en do mineur, op. 68. On peut écouter une réalisation par Michel Tabachnik en suivant ce lien : http://youtube/ogEUlX8paR4

3. Dans cette pièce, il y a effectivement plusieurs niveaux de compréhension de l'entrée en scène des interprètes, à la fois par rapport à l'instrument et par rapport au plateau. Voir à ce propos : http://youtube/vxyrwahgq7o, consulté le 29 octobre 2012

4. Expression attribuée à l'époque par les critiques musicaux (mais surtout par Hans von Wolzogen) à propos des motifs mélodiques récurrents que Wagner utilise pour construire ses opéras. Un Leitmotiv (ou "motif dirigé ») possède moins une fonction dramatique lorsqu'il est pris isolément pour lui-même (auquel cas, il relèverait des mêmes procédés que l'on trouve dans le mélodrame par exemple) que lorsque l'on considère l'ensemble de ses apparitions et de ses 
motifs dérivés, dans le travail de transformation et de variation dont il est l'objet et qui intervient au cours de l'œuvre en accompagnant l'évolution dramatique. Cette cohérence des moyens dramatiques et musicaux s'inscrit dans le projet wagnérien d'art total, Gesamtkunstwerk.

5. Il s'agit de la fosse d'orchestre, située plus ou moins sous le plateau de scène lorsque l'on joue un opéra, et qui est plus ou moins recouverte par le proscenium.

INDEX

Mots-clés : musique, musicien, geste 